\title{
Synthesis and Properties of Thermo-sensitive Poly(N-propionyl-L-aspartic acid-co-1,3-propylene glycol)
}

\author{
Jihua Song ${ }^{\star 1}$, Meng Wang ${ }^{1}$, Akoda Komlan Elom ${ }^{1}$ Chang Zheng ${ }^{1}$, Zhihui Fan ${ }^{1}$ \\ ${ }^{1}$ College of Chemistry \& Environmental Science, Hebei University, Baoding 071002, China \\ Tel: +86-0312-5971137; Fax: +86-0312-5079525; E-mail: 1254336934@qq.com
}

\begin{abstract}
Keywords: Thermo-sensitive Polymer, Upper Critical Solution Temperature(UCST), L-aspartic acid, Cell Toxicity

Abstract: In this paper, a novel poly( $N$-propionyl- $L$-aspartic acid-co-1,3-propylene glycol) (PPAP) was successfully synthesized with $L$-aspartic acid and 1,3-propylene glycol. The chemical structure of poly( $N$-propionyl- $L$-aspartic acid-co-1,3-propylene glycol) was characterized by FTIR and ${ }^{1} \mathrm{H}$ NMR spectrum. The UV measurements indicated that PPAP showed a reversible phase transition and its UCST was tunable via changing the ratio of methanol(ethanol) and water. The viability of HeLa cells was found to be higher $80 \%$ after 24,48 and $72 \mathrm{~h}$ incubation in the different concentrations $(0.01,0.1,1.0,10$, and $100 \mu \mathrm{g} / \mathrm{mL})$ of PPAP. In summery, the experimental results showed that the synthesized polymer had excellent thermo-sensitivity and biocompatibility.
\end{abstract}

\section{Introduction}

With the development of modern biomedical engineering, the role of temperature responsive polymer become more and more significant as a kind of smart material. In recent years, a variety of medical polymer materials with low toxicity, high efficiency and controllable release properties have been prepared. Polymers which prepared from aspartic acid have not only good water solubility but also biocompatibility and biodegradation. In addition, the final degradation products are harmless to the environment. Researchers realized that the aspartic acid materials have a wide range of potential applications in biomedical field such as drug delivery [1-2], tissue engineering [3-4], bio-separation [5-6], drug release, artificial skin and so on.

Smart polymers which can response to external stimuli such as temperature, $\mathrm{pH}$, electric field and magnetic are environmentally sensitive polymers. Currently, responsive polymers have been widely used in the field of drug delivery. Certainly, preparation and investigation of this polymer must be satisfied with the following three requirements: good thermo-sensitivity, biological degradability and compatibility. In this study, a new poly( $N$-propionyl- $L$-aspartic acid-Co-1,3-propylene glycol) was prepared and the experimental results showed the polymers had good biocompatibility and thermo-sensitivity.

\section{Experiment}

Materials. $L$-aspartic acid, 1,3-propylene glycol, methanol $(\mathrm{MeOH})$, ethanol $(\mathrm{EtOH})$, propionic anhydride and ethyl ether were purchased from Kermel chemical reagent (Tianjin, China). Oxalyl chloride and formic acid were purchased from Tianjin Huadong Reagent Factory. Acetone need to be refined. Other reagents and solvents were analytical grade and used without further purification.

Preparation of poly( $N$-propionyl- $L$-aspartic acid-co-1,3-propylene glycol). Firstly, $N$-propionyl- $L$-aspartic acid was synthesized with $L$-aspartic and propionic anhydride. Secondly, $N$-propionyl- $L$-aspartic acid acyl chloride was prepared with $N$-propionyl- $L$-aspartic acid and oxalyl chloride. Thirdly, $N$-propionyl- $L$-aspartic acid acyl chloride $(1 \mathrm{~g})$ and steamed 1,3-propylene glycol $(0.3366 \mathrm{~g})$ were added to a test tube with electromagnetic stirrer. Then put it in the oil bath and slowly heated to $80{ }^{\circ} \mathrm{C}$ for polymerization 12 hours. When there was no flow phenomena or the concentration of mixture unchanged in the test tube, the reaction was stopped. After returning to room temperature, the polymer was dissolved with methanol. Then the solution was dropped into ether in a beaker under stirring to get the target products. After repeating the above operation several times, the purified poly( $N$-propionyl- $L$-aspartic acid-co-1,3-propylene glycol) (PPAP) was 
obtained. The polymer was dried under vacuum at 40-50 ${ }^{\circ} \mathrm{C}$ for about $24 \mathrm{~h}$. The Fig. 1 showed the preparation of poly( $N$-propionyl- $L$-aspartic acid-CO-1,3-propylene glycol).<smiles>CCC(=O)NC(CC(=O)Cl)C(=O)NC(CC(=O)OCCCOC(C)(C)C)C(=O)C(C)(C)CCO</smiles>

Fig. 1 Preparation of poly( $N$-propionyl- $L$-aspartic acid-co-1,3-propylene glycol)

\section{Results and Discussion}

Structural Characterization of poly( $N$-propionyl- $L$-aspartic acid-co-1,3-propylene glycol). The FTIR and ${ }^{1} \mathrm{H}$ NMR spectrum of poly ( $N$-propionyl- $L$-aspartic acid-co-1,3-propylene glycol) were shown in Fig. 2 and Fig. 3. In the FTIR spectrum, the stretching vibration of the amide linkage $(\mathrm{N}-\mathrm{H})$ in the amide group was recorded at $3366 \mathrm{~cm}^{-1}$. The peak at $1732 \mathrm{~cm}^{-1}$ was attributed to the vibration absorption of carbonyl $(\mathrm{C}=\mathrm{O})$ in the ester group. The peak at $1530 \mathrm{~cm}^{-1}$ was assigned to the bending vibration of the amide linkage $(\mathrm{N}-\mathrm{H})$ in the amide group. The peak at $1283 \mathrm{~cm}^{-1}$ was attributed to the outer surface vibration absorption peak of $\mathrm{C}-\mathrm{N}$ in the amide group and indicated the presence of the propionyl group. The stretching vibration for C-H in PPAP appeared at $2798 \mathrm{~cm}^{-1}$. These results suggested that the synthesis of poly( $N$-propionyl- $L$-aspartic acid-co-1,3-propylene glycol) was successful.

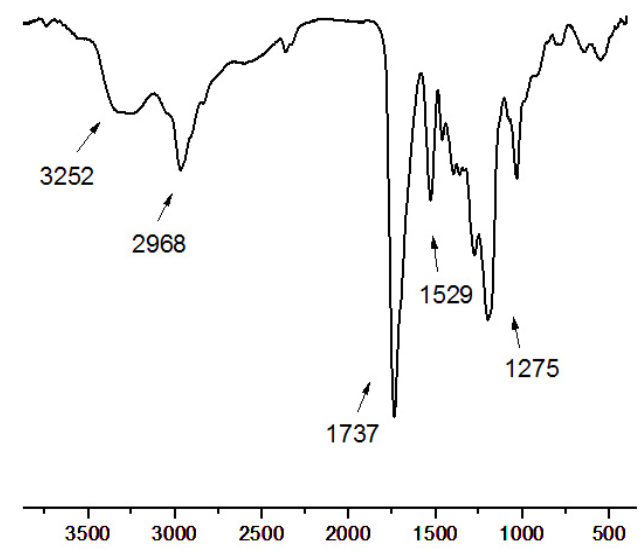

Fig. 2 FTIR Spectrum of PPAP

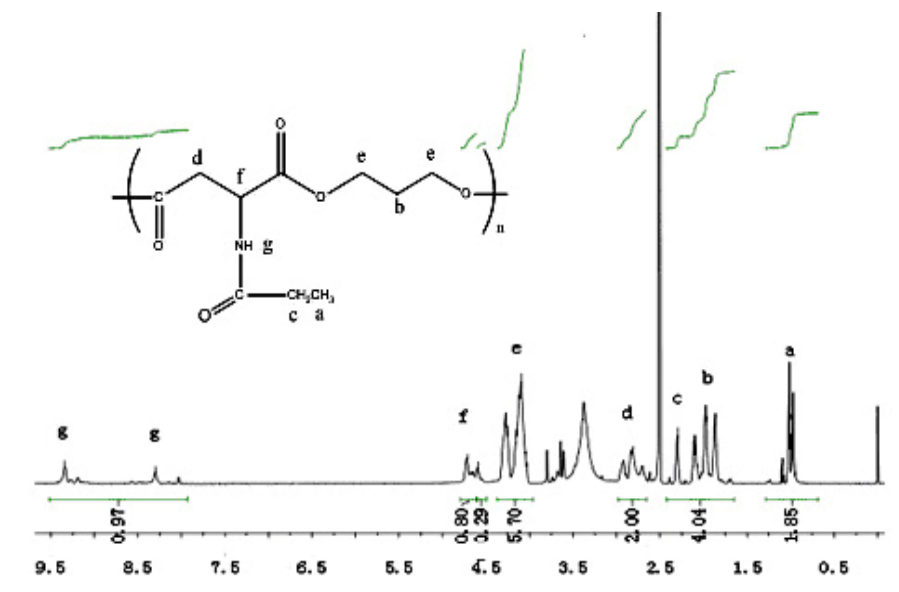

Fig. $3{ }^{1} \mathrm{H}$ NMR Spectrum of PPAP

The ${ }^{1} \mathrm{H}$ NMR spectrum of poly ( $N$-propionyl- $L$-aspartic acid-co-1,3-propylene glycol) in deuterated DMSO was shown in Fig. 3. The absorption peaks at 0.94-1.00 ppm were ascribed to the methyl proton peak (a). The signal at $2.30 \mathrm{ppm}$ was corresponding to the methylene peak (c). The peaks at $1.97 \mathrm{ppm}$ and 4.09-4.25 ppm were assigned to the methylene protons (respectively $\mathrm{b}$ and e ) in propylene glycol. The signal at 4.59-4.71 ppm was ascribed to the proton from the methine of -CH-NH-. The peak at $2.81 \mathrm{ppm}$ was corresponding to the proton of - $\mathrm{CO}-\mathrm{CH}_{2}$ - group. Additionally, the peaks at $8.29 \mathrm{ppm}$ and $9.35 \mathrm{ppm}$ were assigned to protons in amide group. According to the above analysis, this structure was fully consistent with the target compound.

Thermo-sensitivity of poly( $N$-propionyl- $L$-aspartic acid-co-1,3-propylene glycol). As shown in Fig. 4, it confirmed the responsiveness of poly( $N$-propionyl- $L$-aspartic acid-co-1,3-propylene glycol) to the external temperature in different solvents. In methanol, the transmittance of polymer aqueous solution gradually became higher and the solution became clear with the increase of temperature. The UCST of PPAP was measured as $30{ }^{\circ} \mathrm{C}$ in the heating run. But with the decrease of temperature, the transmittance became lower and the solution became turbid. The UCST of PPAP 
was $27{ }^{\circ} \mathrm{C}$ in the cooling run. In ethanol, it could be observed that the UCST of PPAP was $30{ }^{\circ} \mathrm{C}$ in the heating run, and the UCST was $33{ }^{\circ} \mathrm{C}$ in the cooling process. The experimental results showed that PPAP had the characteristics of temperature-sensitivity and the phase transition was reversible.

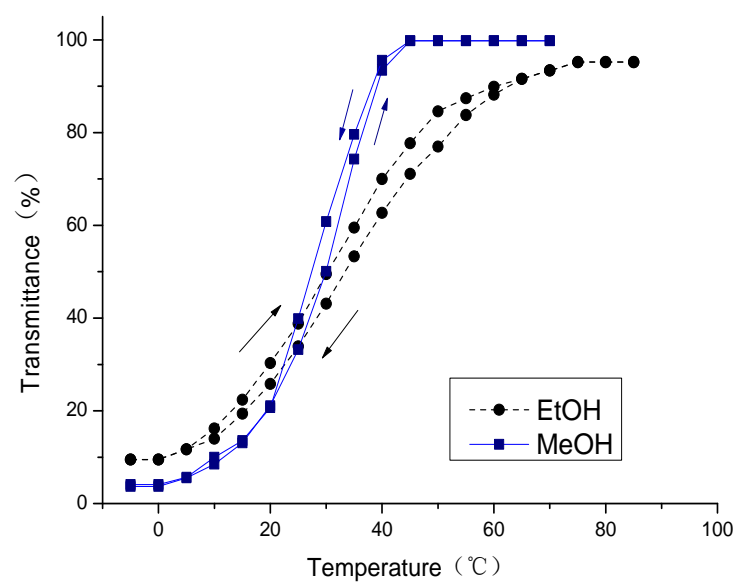

Fig. 4 Reversible phase transition of PPAP in $\mathrm{MeOH}$ and EtOH versus temperature during a heating and cooling run
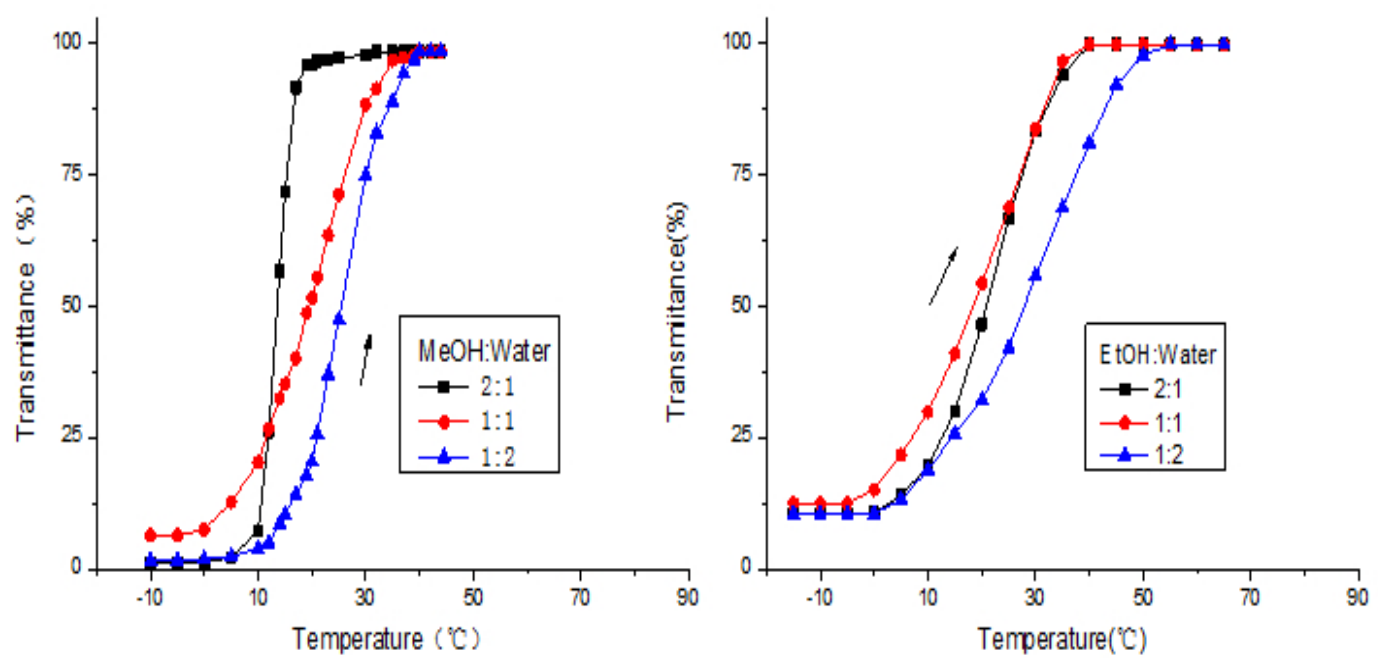

Fig. 5 The phase transition profiles of PPAP in $\mathrm{MeOH} /$ water and EtOH/water

For the UCST of polymer, it was affected by the mixed solvent. In addition, there was a process that the UCST firstly decreased and then increased, which showed the "turn back phenomenon" with the increase of alcohol content. As shown in Fig. 5, when the quality ratio of methanol and water was $0.5: 1,1: 1$ and 2:1 in the mixed solution of methanol and water, the corresponding UCST was $25{ }^{\circ} \mathrm{C}, 19{ }^{\circ} \mathrm{C}$ and $13{ }^{\circ} \mathrm{C}$. For the ethanol/water mixed solution, the corresponding UCST was $28{ }^{\circ} \mathrm{C}, 18{ }^{\circ} \mathrm{C}$ and $20{ }^{\circ} \mathrm{C}$, respectively. At the same time, the temperature range of phase transition became smaller with the increase of the content of alcohol in the mixed solution.

Cytotoxicity evaluation for poly( $N$-propionyl- $L$-aspartic acid-co-1,3-propylene glycol). In this study, the cell cytotoxicity of poly( $N$-propionyl- $L$-aspartic acid-co-1,3-propylene glycol) was evaluated using MTT method. HeLa cells were cultured in the different concentrations $(0.01,0.1$, 1.0, 10, and $100 \mu \mathrm{g} / \mathrm{mL}$ ) of PPAP for 24, 48 and $72 \mathrm{~h}$, respectively. As shown in Fig. 6, the cell viability was all higher $80 \%$ after $24 \mathrm{~h}, 48 \mathrm{~h}$ and $72 \mathrm{~h}$ incubation in the different concentrations of PPAP. The experimental data suggested that PPAP had highly biocompatible and no cytotoxicity against HeLa cells. Namely, Hela cells can survive over a relatively long exposure time to PPAP materials. 


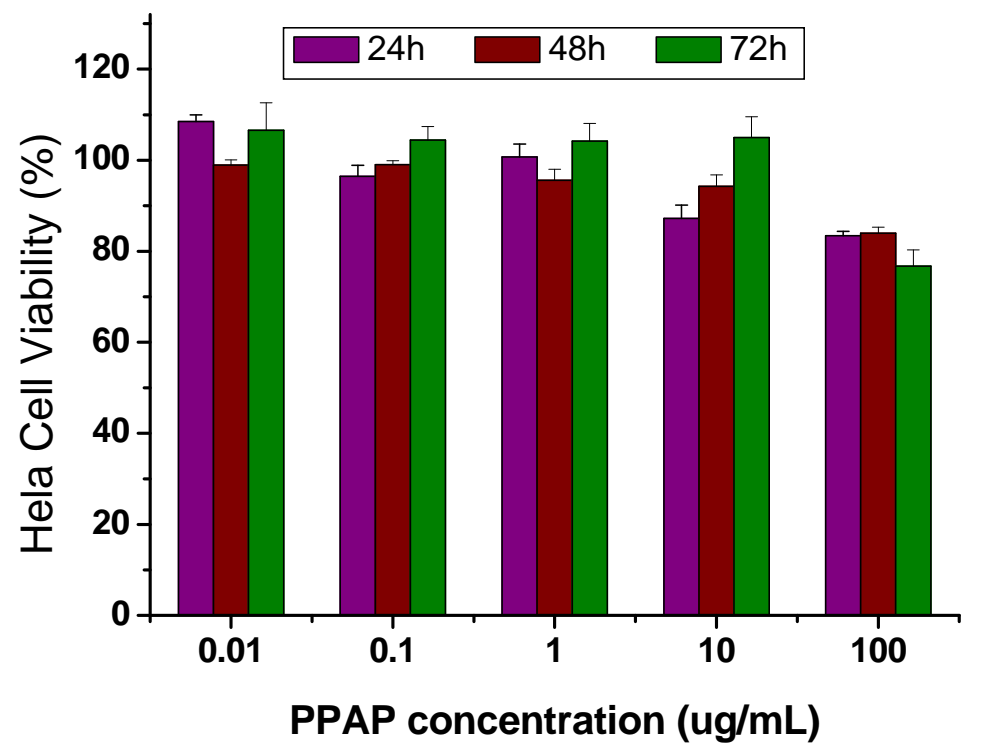

Fig. 6 Viability of HeLa cells as a function of PPAP concentration and time

\section{Conclusions}

In this paper, we successfully synthesized a novel poly( $N$-propionyl- $L$-aspartic acid-co-1,3-propylene glycol) (PPAP). The experimental results indicated that PPAP had the reversible phase transition behavior to the external temperature. The upper critical solution temperature (UCST) of PPAP was regulated by changing the solvent. In addition, the UCST was also controlled by changing the number of hydrophilic and hydrophobic groups in polymer chains. MTT assessment method was used to study the cytotoxicity of PPAP which showed good biocompatibility, and the results suggested that PPAP had no cytotoxicity against HeLa cells. Based on the characteristics of good biocompatibility and thermo-sensitivity, PPAP provided a good security guarantee for its practical application in biomedical field.

\section{Acknowledgements}

This work was supported by Hebei Natural Science Foundation of China (B2008000573)

\section{References}

[1] B. Tasdelen, N.K. Apohan, O. Güven and B.M. Baysal: Polym. Adv. Technol. Vol. 15 (2004), p. 528

[2] S.V. Ghugare, P. Mozetic and G. Paradossi: Biomacromolecules. Vol. 10 (2009), p. 1589

[3] A. Galperin, T.J. Long and B.D Ratner: Biomacromolecules. Vol. 11 (2010), p. 2583

[4] W.I. Sopita, K.T.N. Thi, S. Wanwipa and V.P. Kwanchanok: J. Appl. Polym. Sci. Vol. 129 (2013), p. 3061

[5] S. Champ, W. Xue and M.B. Huglin: Polymer. Vol. 42 (2011), p. 6439

[6] R.B. Fong, Z. Ding, A.S. Hoffmanl and P.S. Stayton: Biotechnol. Bioeng. Vol. 79 (2002), p. 271 